\title{
DUE/ER/40438..T3
}

CONTINUATION OF DOE GRANT NO. DE-FG02-88ER49738 : . .

THE UNITED STATES DEPARTMENT OF ENERGY

DOE/ER/ $40438--T 3$

DE91 005791

\section{Development of a Hydrogen and Deuterium Polarized Gas Target \\ for Application in Storage Rings}

\section{FROM}

W. Haeberli, Principal Investigator

Department of Pliysics, College of Letters and Science

University of Wisconsin, Madison, Wisconsin 53706

The Regents of The University of Wisconsin

750 University Avenue

Madison, Wisconsin 53706

\section{DISCLAIMER}

This report was prepared as an account of work sponsored by an agency of the United States Government. Neither the United States Government nor any agency thereof, nor any of their employees, makes any warranty, express or implied, or assumes any legal liability or responsibility for the accuracy, completeness, or usefulness of any information, ar paratus, product, or process disclosed, or represents that its use would not infringe privately owned rights. Reference herein to any specific commercial product, process, or service by trade name, trademark, manufacturer, or otherwise does not necessarily constitute or imply its endorsement, recommendation, or favoring by the United States Government or any agency thereof. The views and opinions of authors expressed herein do not necessarily state or reflect those of the United States Government or any agency thereof.

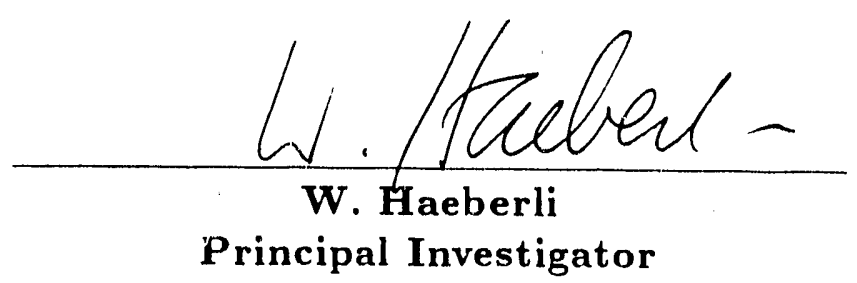




\section{INTRODUCTION}

The present report contains the progress report for the second year of the 3-year budget period, and proposes work for the third year. Progress has been made on the two major components of the project, the tests of storage cells for polarized atoms under various operating conditions, and the construction of a new atomic beam source which conforms to the high vacuum requirements of storage rings.

Early in the year we had to make difficult decisions on how to apply the limited resources we had available most effectively. With only one person working on the construction of the new atomic beam source, it could be foreseen that completion of this project would take unreasonably long. Work at other laboratories indicates that about a 10 man-year effort is needed for this type of project. We were able to alleviate the problem of manpower shortage by "creative financing". We hired a second Research Assistant (Andrew Roberts) to work with our Physicist (Tom Wise) on the atomic beam source. In addition, during the summer and part of the semester we had the help of an undergraduate student who was paid by the NSF Undergraduate Research Participation Program. Funding for the second Research Assistant was made pussible by making a number of small sacrifices: the Research Assistant responsible for the tests of storage cells (Scott Price) was participating for three months in a NSF-funded experiment at the Indiana Storage ring, so he would get familiar with the everitual applications of his research on storage cells in storage rings. Tom Wise is consulted now and then for half a day at a time by the users of the tandem accelerator for his expertise in polarized ion sources, and to alleviate our funding problem we requested a contribution to his salary from the NSF budget. Finally, Andrew Roberts runs one 8 liour shift a week as a tandem operator, thus part of his pay was also charged to other funds. Overall, these compromises have been highly beneficial to our DOE supported research. Overall the effect has been to somewhat slow down the storage cell work of Scott Price and to significantly speed up the assembly and debugging of the new atomic beam apparatus. 


\section{PROGRESS REPORT}

\subsection{New Results of Storage Cell Tests}

\section{a) Introduction}

We have completed a series of tests on new storage cell surfaces using the improved test method described below.

The test method, which has been described before ${ }^{1}$, consists of passing a $50 \mathrm{keV}$ beam of $\mathrm{D}^{+}$ions through the polarized target gas, where some of the ions pick up a polarized electron and turn into polarized $50 \mathrm{keV}$ neutral deuterium atoms, $\mathrm{D}^{\circ}$. The polarization of the $D^{\circ}$ is subsequently detected by the anisotropy of ${ }^{3} \mathrm{H}(\mathrm{d}, \mathrm{n})^{4} \mathrm{He}$ neutrons produced by the $D^{\circ}$ incident on a tritiated titanium target, using neutron detectors at $0^{\circ}$ and $90^{\circ}$ with respect to the magnetic field axis at the target. The anisotropy of the neutrons from the ${ }^{3} \mathrm{H}(\mathrm{d}, \mathrm{n}){ }^{4} \mathrm{He}$ reaction is only $8 \%$ for a completely polarized target. Rather than comparing the neutron count rates to the rates measured with an unpolarized target, the effect can be doubled by reversing the tensor polarization of the deuterons. The polarization reversal is accomplished by the "sudden field reversal method" originally proposed by Sona ${ }^{2}$ for polarization enhancement in Lamb-Shift polarized ion sources. The efficiency of the polarization reversal is about $90 \%$ and is checked in a separate test measurement at the beginning and end of every cell polarization run.

\section{b) Fluorinated Polymer Coatings}

The new system allows measurements of target polarization vs. temperature of the cell walls down to $20 \mathrm{~K}$. Cooling of the cell has the advantage of increasing the target gas density in proportion to $T^{1 / 2}$, but has the potential disadvantage that the increased residence time of the atoms on the wall may lead to spin relaxation. The curves presented in Figures 1 and 2 show polarization vs. temperature for various coated and uncoated surfaces. The curves in these figures are based on the idea that an atom sticks on the cell wali for a time dependent upon $\exp \left(E_{b} / k T\right)$, where $E_{b}$ is the binding energy for the atom on the surface, and that the polarization of atoms which are in contact with the wall for a time $t$ decays like $\exp \left(-t_{t} / \tau_{R}\right)$, where $\tau_{R}$ is the relaxation time. 
Teflon FEP 120: Results for the temperature dependence of Teflon FEP 120 coated. cells (Fig. 1 a) show a loss in polarization below about $130 \mathrm{~K}$. For a cell with an averarge of 380 wall collisions, half of the polarization is lost at $\mathrm{T}_{1 / 2}=85 \mathrm{~K}$. As expected, $\mathrm{T}_{1 / 2}$ decreases when one reduces the number of wall collisions. We have also tested other wall materials vs. temperature, but have not discovered any wall materizals that would allow cooling below about $100 \mathrm{~K}$ without significant loss in polarization.

Teflon TE3170 PTFE: Wall coatings must be able to survive high temperature bake out procedures if thev are to be used in the UHV environment of accelerator rings. We tested the PTFE (polytetrafluoroethylene) form of Teflon which has a higher melting point and service temperature as compared to Teflon FEP 120 and found that it has nearly the same polarization vs. temperature characteristics. This is an improvement in that we can expect a more resistant coating.

Fomblin: The useful lifetime of polymer coatings in the environment of a storage ring is not yet known, but may be compromised by synchrotron radiation damage. One solution to the damage resistance problem is to find a wall coating that can be renewed or repaired in situ. While a renewable or renewing solid cell wall poses a number of difficult technical problems, a liquid coating with a temperature dependent viscosity might prove to be an option: one can imagine cooling a cell for use, then warming the liquid to anneal the damaged surface.

Fomblin (a perfluoropolyether) nil has been used successfully in cold neutron experiments as a wall surface coating ${ }^{3}$. One Fomblin oil (YVAC 18/08) has a vapor pressure of $2 \times 10^{-8}$ Torr at $293 \mathrm{~K}$. This coating has proven to be one of the best in terms of polarization and reliability (Fig. 1b). The high vapor pressure problem can be avoided by cooling the cell or by using a grease form of Fomblin with a much higher viscosity and lower vapor pressure $\left(5 \times 10^{-13}\right.$ Torr at $\left.293 \mathrm{~K}\right)$. Unfortunately, Fomblin grease does not perform as well as the oil (Fig. 1b) in a weak holding field. Fluorinated Drifilm: Normal Drifilm ( $\mathrm{CH}_{3}$ groups on the surface, Si-O-Si backbone) has been successfully used for extended periods in an electron ring at Novosibirsk ${ }^{4}$ in the presence of a magnetic field strong enough to decouple the nuclear spin from the electron. In a weak magnetic field, our measurements yield lower polarization for Drifilm than for Teflon (Fig. 1c). We tested the properties of a fluorinated 

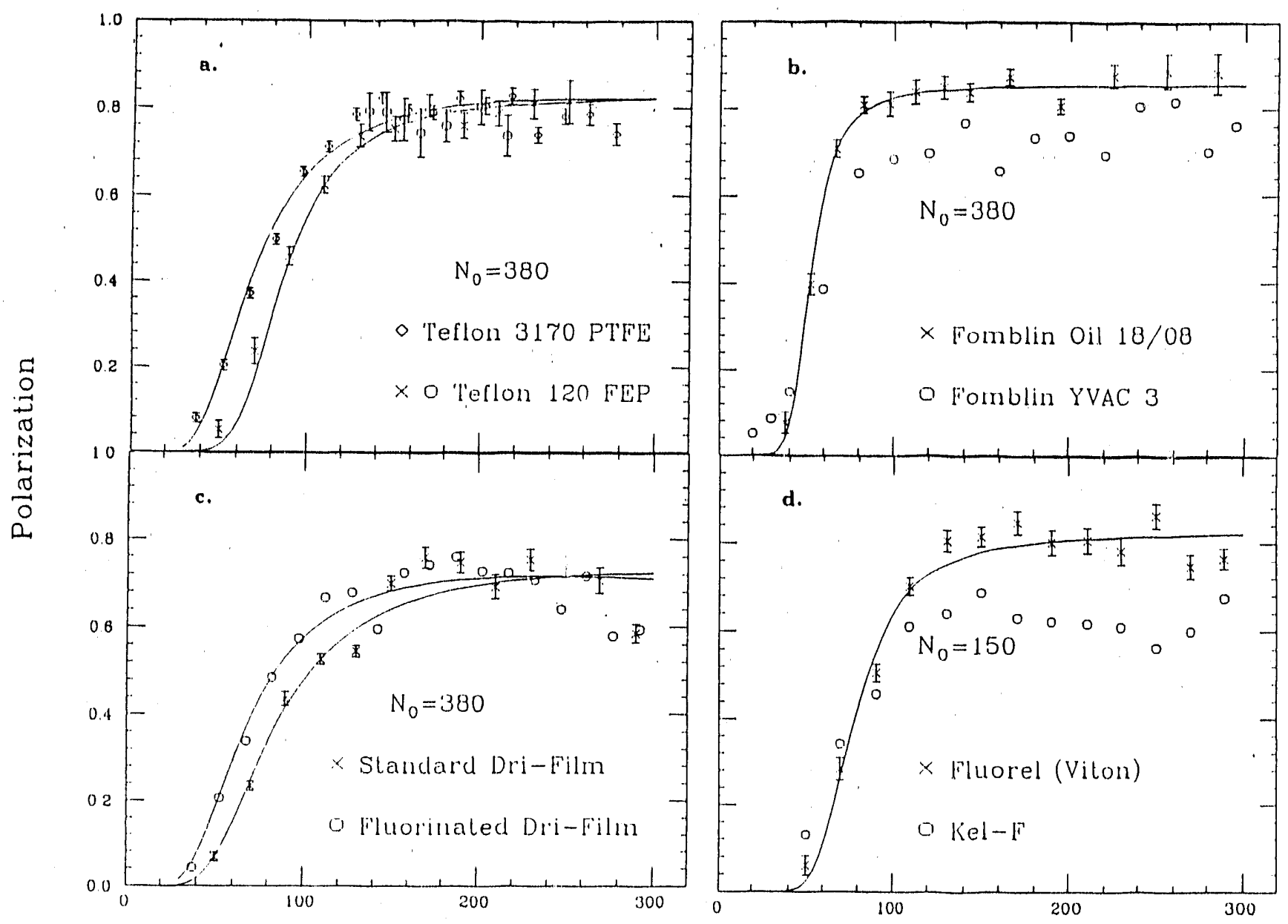

T'emperature (K)

FIG. 1. Polarization of hydrogen atoms in storage cells with different wall coatings as a function of cell wall temperature.

version of Drifilm, which has $\mathrm{CF}_{3}$ groups on the surface, and may be more resistant to radiation. Although the polarization for the fluorinated Drifilm proved to be no better, an unexpected dividend was that the $\mathrm{T}_{1 / 2}$ moved down to $60 \mathrm{~K}$ for a 380 bounce cell as compared to $80 \mathrm{~K}$ for normal Drifilm. One can cool a fluorinated Drifilm cell thus increasing the density of the target gas without sacrificing as much polarization as compared to normal Drifilm.

Viton: This low outgassing rate elastomer used for vacuum seals can be applied to storage cell walls in an organic solvent form called Fluorel (Fig. 1d). 


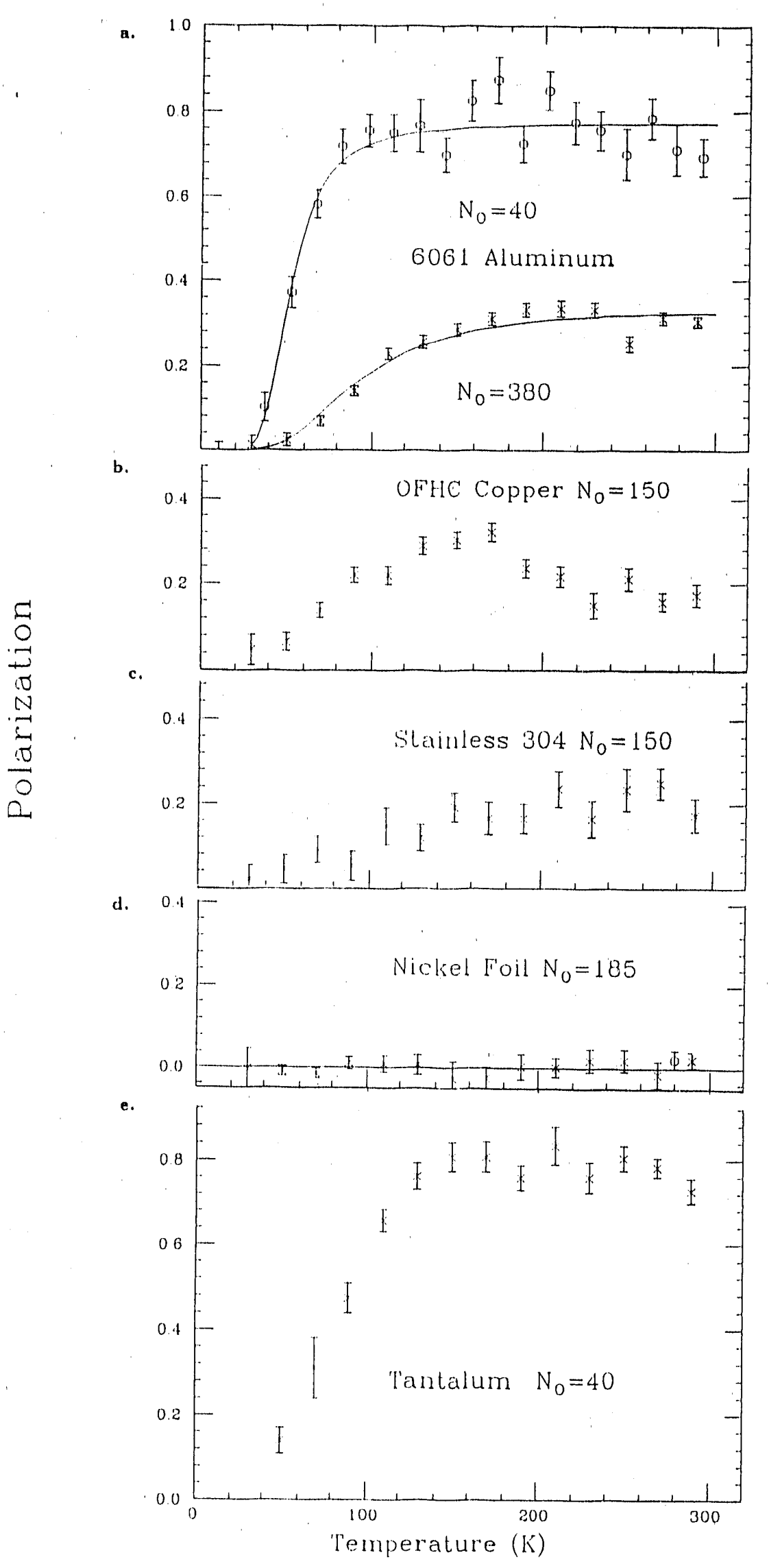

FIG. 2. Polarization of hydrogen atoms in storage cells without applying wall coatings. 
Kel-F: Kel-F (a chlorotrifluoroethylene polymer) presents good vacuum compatibility in bulk material form. The outgassing is low and comparable to Teflon FEP 120. The organic solvent coating form of this resin ( $\mathrm{Kel}-\mathrm{F} 800)$ was used to coat cells. A 40 bounce rell performed quite well, whereas a 380 bounce cell shows serious depolarization (See Fig. 1d).

\section{c) Uncoated Metals}

Some of the best cell wall coatings, those that have proven to inhibit depolarization and recombination in a weak field, have been fluorinated polymers: Teflon, Fomblin and Viton. The useful lifetime of these coatings in the environment of a storage ring is not yet known, but may be compromised by synchrotron radiation damage. Cell wall surfaces of uncoated inetal may present significant advantages in ihis respect, even if covered with a film of adsorbed gas. Results for various metal cells are shown in Fig. 2.

Most uncoated metals have high temperature plateau polarization values that are well below the uncoated aluminum (which has a natural oxide layer on the surface). For a 150 bounce geometry aluminum cell the polarization value at the high temperature platean is about $45 \%$.

Tantalum (e) is an exception in that it matches aluminum's performance in inhibiting depolarization. Like aluminum, this metal can withstand high bake-out temperatures necessary for accelerator ring operation and can be fashioned into thin foils. It needs to be demonstrated that such bake out does not cause alteration of the surface which would change the cell wall depolarization properties. One disadvantage for Ta is the large stopping power and high mass density, thus making it unsuitable for applications in which low energy recoils are to be detected.

\section{d) Reproducibility of Wall Properties}

Since wall depolarization is a surface effect, reproducibility of surface conditions is important if one compares cells of different geometry and different temperatures. Thus a major effort was made to check the reproducibility of all recent and earlier measurements. Consequently measurements are always repeated with at least one other cell of the same geometry, and the hysteresis in the temperature dependence is checked by making measurements with decreasing and with increasing temperatures. 


\subsection{Strong Field Tests}

Tests in the presence of a strong magnetic field over the target cell must be carried out for two reasons: (1) in electron rings, the periodic electromagnetic pulse of the circulating electrons will depolarize the target unless a magnetic field is applied which decouples the nuclear spin from the electron spin. The field strength has to be chosen to be between spin flip resonances; (2) some of the surfaces we have studied (e.g. aluminum with a natural oxide layer) show significant loss in polarization compared to a Teflon coated cell, but have advantages in other respects: excellent vacuum compatibility and a well-known high resistance to radiation damage. We propose to study whether large target polarization is obtained with oxidized aluminum in a 380 bounce geometry cell if a magnetic field is applied to decouple the nuclear spin from the electron spin. In addition, we will be interested to find out if the presence of a strong magnetic field avoids the depolarization which, in a weak magnetic field, we observed for all surfaces when the wall is cooled below about $100 \mathrm{~K}$.

These tests require significant changes in our apparatus. Besides the changes required to accommodate magnetic field coils inside the UHV chamber, the entire de iection method has to be changed. In the present method we detect the electron polarization of the target atoms, which in a weak field reflects the nuclear polarization. In the presence of a B-field strong enough to decouple the spins of the electron and the proton, the nuclear polarization itself has to be detected. This will require that the atoms in the storage cell be ionized and that the polarization of the ions be measured. We plan to accomplish this by using deuterium as the target atoms. The deuterons are to be accelerated to some $100 \mathrm{keV}$, and the alignment of the delaterons is to be measured by the ${ }^{3} \mathrm{H}(\mathrm{d}, \mathrm{n})^{4} \mathrm{He}$ reaction as it is done at present. The ionization of the deuterium can be achieved in one of two ways: (a) use an electron bombardment ionizer external to the cell to ionize the deuterium atoms emerging from the cell, or (b) pass an ionizing beam through the cell, thus ionizing the deuterium atoms within the cell, and extracting the ions using an electrode at one end of the cell.

a) Ionization external to the storage cell: A strong field ionizer of the Glavish type ${ }^{5}$ (which we have on hand) can be placed collinear with the cell tube axis in order to ionize the intercepted portion of the deuterium atoms leaving one end of the cell. The principal difficulty of this method is that atoms emerge from the storage cell over a wide range of angles, so that a significant fraction of the atoms hit the inner 
wall of the ionizer. Thus, many of these atoms will be depolarized before they reach the ionization volume, and the measured nuclear polarization of the extracted ions no longer reflects the polarization of the atoms in the storage cell. The problem can be avoided by collimating the deuterium atoms emerging from the cell with apertures and placing the ionizer farther away from the cell. The collimated deuterium atoms would then be jonized without first colliding with the inner wall, removing unwanted background but severely reducing the flux of deuterium atoms entering the ionizer. Using the known ionization efficiency of the Glavish ionizer, one can estimate the number of $\mathrm{D}^{+}$ions extracted when the atoms emerging from the storage cell are collimated by a $1 \mathrm{~cm}$ diameter collimator a distance $10 \mathrm{~cm}$ from the exit of the storage cell. Of the $3 \times 10^{15}$ atoms emerging from one end of the storage cell, $3.8 \times 10^{13}$ atoms enter the ionization volume. The efficiency of the ionizer being $2 \times 10^{-3}$, about $7.5 \times 10^{10} \mathrm{D}^{+}$ions or $12 \mathrm{nA}$, can be extracted from the strong field ionizer. If these ions are accelerated to $100 \mathrm{keV}$, the corresponding count rate is about $300 \mathrm{~Hz}$. This low signal rate and a potentially high background rate make this option unattractive.

b) Ionization internal to the storage cell: We presently pass a $50 \mathrm{keV}$ ion beam through the storage cell to measure the target polarization in a weak magnetic field. Thus we are already producing ions inside the storage cell, and it would seem a simple matter to extract these ions and accelerate them onto a tritium target. On close inspection this turns out to be difficult because of the transverse momentum which the ion receives in the collision process. Measurements of the transverse momentum transfer have been reported for ionization of Argon by a $75 \mathrm{keV}$ beam of Argon ${ }^{6}$. The results show that about $80 \%$ of the ions formed have a transverse energy above $1 \mathrm{eV}$. The question is whether these ions can be extracted before they hit the inner wall of the storage cell. In the presence of an axial magnetic field, the ions will spiral around the magnetic field lines, until they eventually reach the end of the tube where they feel the strong electric ficld of the extraction electrode. The radius of curvature of a $1 \mathrm{eV}$ ion in the magnetic field needed for our tests $(50 \mathrm{mT})$ is $0.4 \mathrm{~cm}$ i.e. most of the ions will hit the wall of the storage cell whose radius is of the order $0.5 \mathrm{~cm}$.

Because of these difficulties, we are preparing equipment to ionize deuterium atoms in the storage cell by electron bombardment. The cross-section for ionization of hydrogen atoms by electron bombardment reaches a maximum of $9 \times 10^{-17} \mathrm{~cm}^{2}$ 
at an electron energy of about $65 \mathrm{eV}$. If $1 \mathrm{~mA}$ of electrons were to make a single pass through the cell of target density $5 \times 10^{12}$ atoms $/ \mathrm{cm}^{2}, 2.6 \times 10^{11} \mathrm{D}^{+}$ions $/ \mathrm{sec}$ are created. If extracted and accelerated to $100 \mathrm{keV}$, a count rate of some 1000 $\mathrm{Hz}$ is expected. The maximum transverse momentum of the deuterium ion after collision, that is consistent with energy and momentum conservation, corresponds to a deuteron energy of $0.027 \mathrm{eV}$. This results in an ion with a radius of curvature of $0.075 \mathrm{~cm}$ in a $50 \mathrm{mT}$ axial magnetic field.

Progress so far: A standard model electron gun has been bench tested in the cell chamber under UHV conditions: $0.65 \mathrm{~mA}$ beam on target at $4500 \mathrm{eV}$ kinetic energy was observed. A high beam intensity model of this same gun has been ordered and further tests will be carried out after receipt of this equipment.

\subsection{Assembly and Optimization of the Atomic Beam Source}

A schematic of the major components of the source is shown in Fig. 3. The vacuum system is shown separately in Fig. 4. Progress in each of the subsystems is discussed in the following paragraphs.

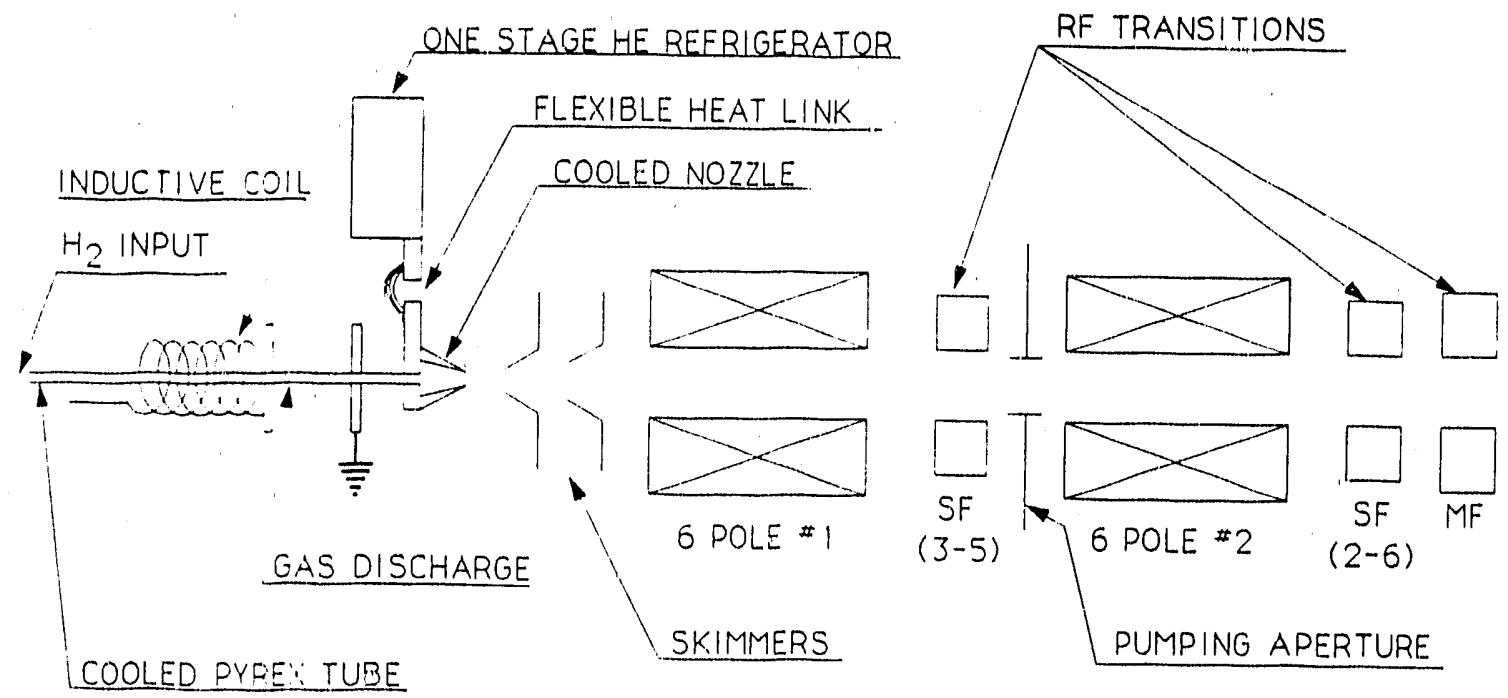

FIG. 3. Schematic of atomic beam source. The vacuum housing is not shown. 


\section{a) Vacuum System:}

The source housing, the pumping systems, and the internal baffles are now assembled. Operation of the system conforms to the design values and permits a $\mathrm{H}_{2}$ throughput as large as 5 mbar $\ell /$ sec. Fig. 4 lists the observed pressures in each of the four chambers. We anticipate improved vacuum in chamber \#4 if the more restrictive pumping aperture originally planned is installed between chambers 3 and 4 . The pressures agree well with the engineering design values.

CHAMBER PRESSURES IN moarS

AT 4 mbar $1 / \mathrm{sec}$ GAS FLOW

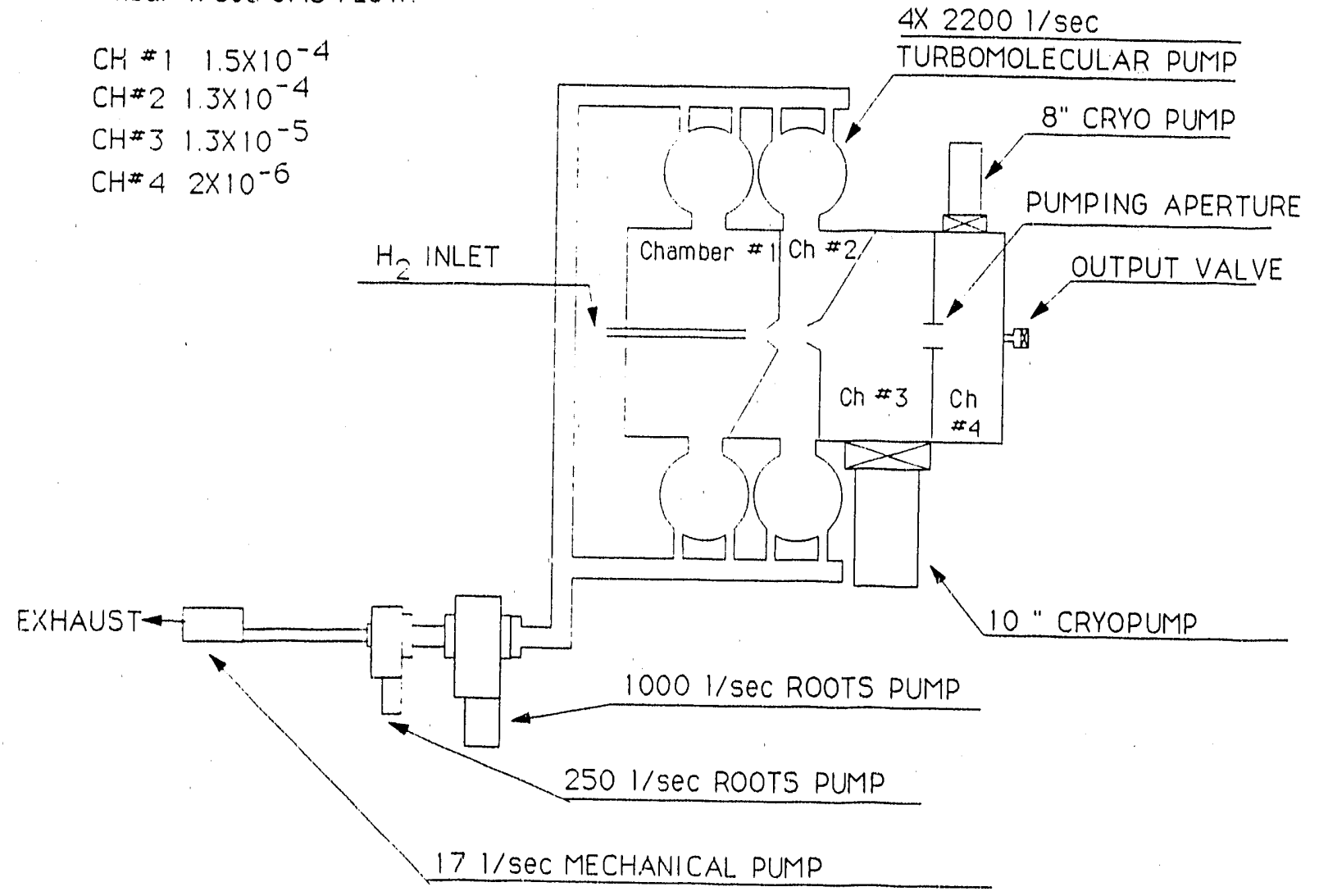

FIG. 4. Differential Pumping System. 


\section{b) RF Dissociator:}

The RF dissociator and RF power system are now operating. The original dissociator, which used inductive coupling, generated a relatively weak discharge with low clegree of dis ociation. This problem was overcome by converting to capacitive coupling. We now observe a strong and stable gas discharge for a wide range of gas flows with nearly zero reflected power. Forward power levels are typically 100-300 W at 18 $\mathrm{MLHz}$.

c) Nozzle Cooling:

It is well known that the beam intensity delivered by atomic beam sources improves when a cooled beam is employed, since cooling the atomic beam increases the acceptance solid angle of the sixpole magnet. We have assembled and tested a nozzle cooling system for this purpose. The system includes a flexible heat link between the nozzle and a one stage helium closed cycle refrigerator. With $300 \mathrm{~W}$ RF discharge power and $4 \mathrm{mbar} \ell / \mathrm{sec} \mathrm{H}_{2}$ flow, we observe a nozzle temperature of $50^{\circ} \mathrm{K}$.

d) Measurements of Degree of Dissociation:

The intensity of the atomic beam depends critically on the degree of dissociation. Typically, a substantial fraction of the $\mathrm{H}_{2}$ molecules either are never dissociated into $\mathrm{H}$ atoms, or $\mathrm{H}$ atoms formed are lost due to recombination. Wall recombination is strongly temperature and surface depenclent. To measure the degree of dissociation we are using a quadrupole mass spectrometer in conjunction with a versatile data acquisition system which we have recently taken into operation. By repeated sampling of the beam it is possible to obtain the degree of dissociation as a function of various parameters such as materials employed, suiface temperature, gas flow rates, RF power, and additions to the $\mathrm{H}_{2}$ gas supplying the discharge. Examples of recent measurements of the degree of dissociation $\alpha$ are shown in Fig. 5. 


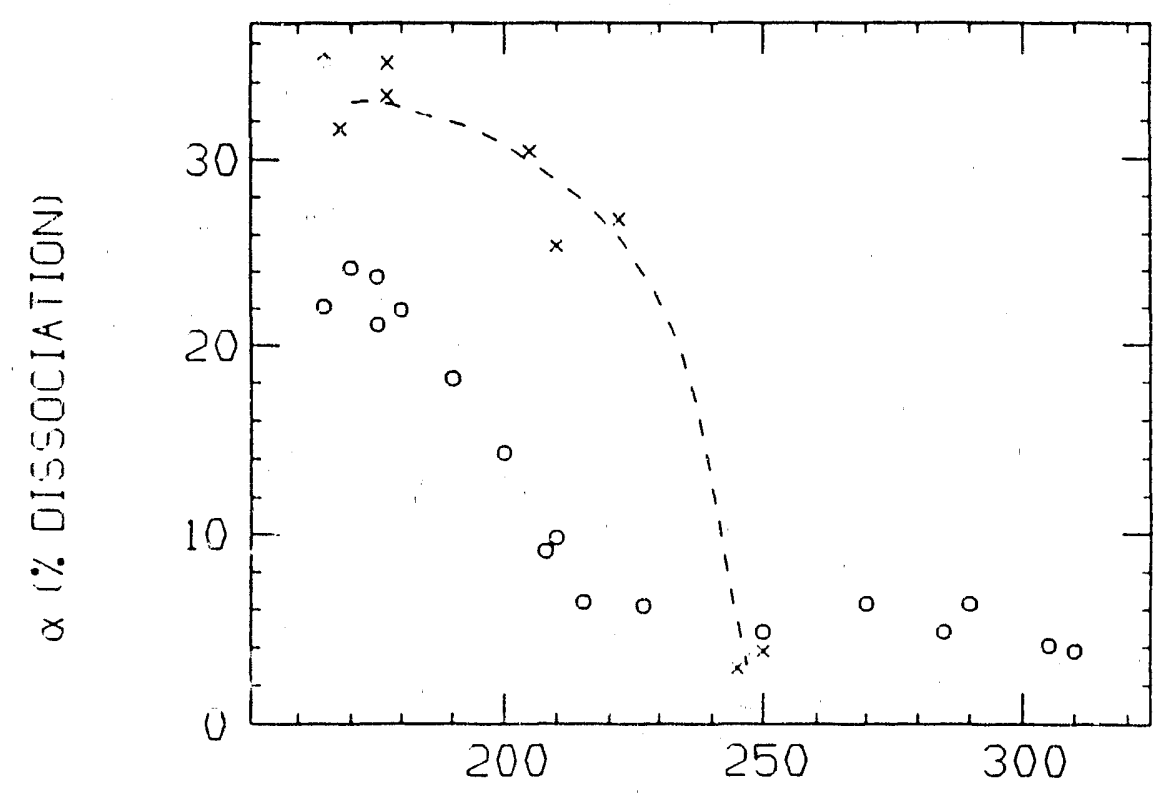

NCZZLE TEMPERATIJRE (KELVIN)

FIG. 5. Degree of dissociation $\alpha$ vs nozzle temperature at $1.5 \mathrm{mbar} \ell / \mathrm{sec}$. gas input. Open circles show $\alpha$ for no water vapor. Crosses show $\alpha$ with water vapor added. The dashed curve guides the eye.

\section{e) Sixpole magnets:}

Magnets continue to be behind schedule. Design of this system was originally intended to be based on experience gained at Heidelberg with permanent magnets on order from Vakuumschmelze. Prolonged delays in magnet delivery and the inherent inflexibility of their design have led us to order magnetic materials and design our own prototype permanent magnets based on a modular system.

The theory of permanent nultiple magnets has been well described by $\mathrm{Halbach}^{7}$. Based on his formulae, we predict a $15 \%$ loss in pole tip strength by reducing the number of magnet elements from 24, as in the Heidelberg magnets, to 12 . This change simplifies construction and greatly reduces magnet cost. We have purchased materials to build three $2.54 \mathrm{~cm}$ long magnet modules with $1.5 \mathrm{~cm}$ bore and two $3.8 \mathrm{~cm}$ long modules with $2.54 \mathrm{~cm}$ bore. An end view of the $2.5 \mathrm{~cm}$ magnet and its housing design is shown in Fig. 6. One useful feature is the ability to change magnet length by adding or removing modules. For purposes of reducing beam attenuation by scattering the modules can be spaced at vurying intervals. The flexibility to 
adjust magnet parameters requires that the $\mathrm{Ne}-\mathrm{Fe}-\mathrm{B}$ magnets be exposed to the atomic hydrogen beam and the ambient vacuum. Unfortunately Ne-Fe-B material is known to be sensitive to $\mathrm{H}$ and $\mathrm{H}_{2}$ exposure. We have nickel plated the pieces to protect them. A plated sample is now being exposed to the hydrogen atomic beam to test the effectiveness of $\mathrm{Ni}$ plating.

One magnet modiule has recently been assembled and magnetic field mapping is under way. First indications are that the field strengih is about $20 \%$ less than the design value.

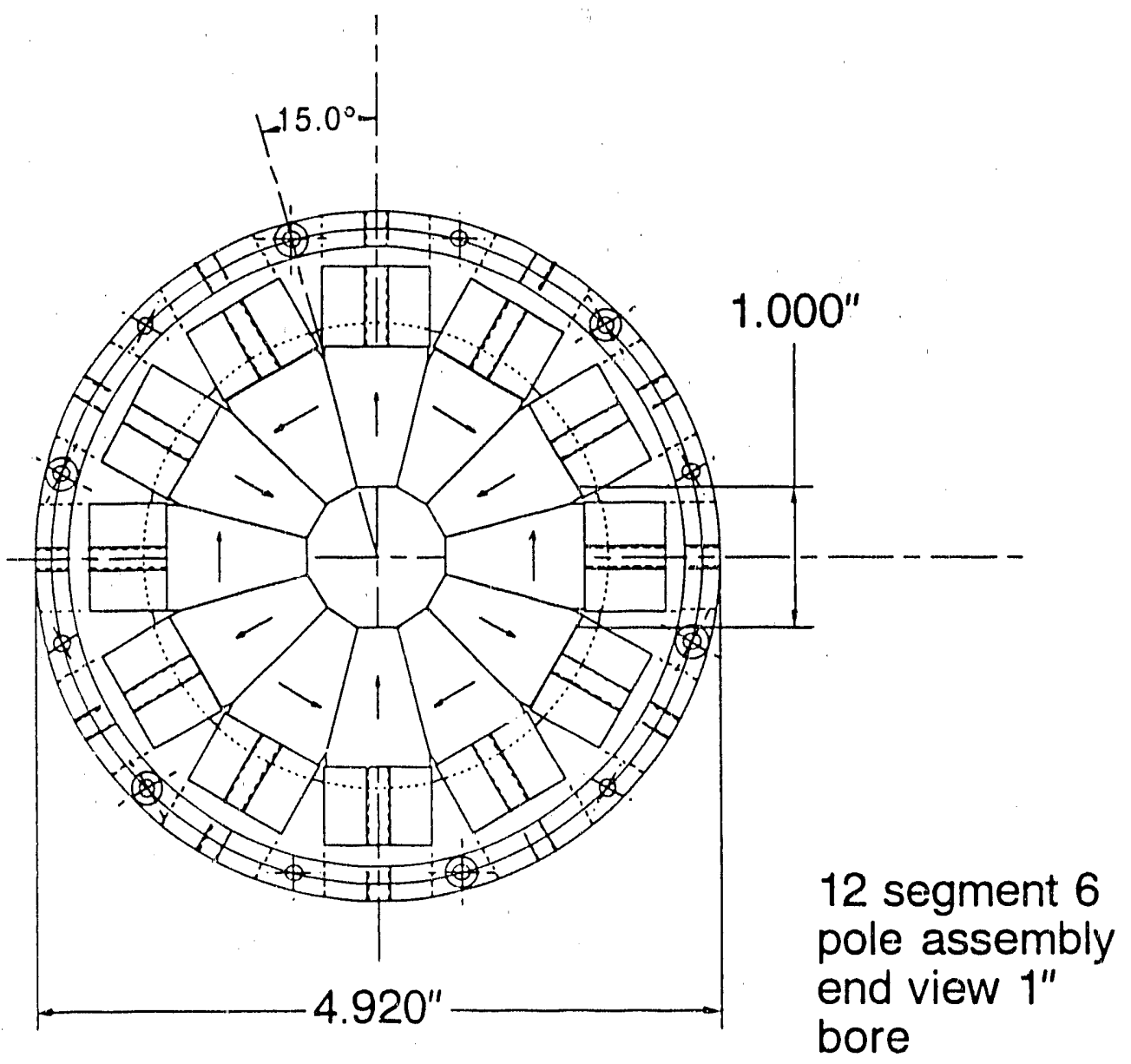

FIG. 6. Detail of 6 pole magnet assembly. The magnet consists of 12 segments which are magnetized in the direction given by the arrows.

\subsection{RF Transitions}

The atomic beam source will be used for production of both hydrogen and deuterium polarized gas targets. Targets placed in hadron storage rings will require a weak holding field over the storage cell because the circulating beam cannot tolerate a large transverse 
magnetic field. For internal polarized gas targets it is desired to achieve the highest possible target density with polarization close to maximum. More precisely one wants to maximize the product $P^{2} I$ where $P$ is the beam polarization and $I$ is the intensity of the atomic beam. For purposes of explanation we describe the case of deuterium in some detail.

Deuterium has six atomic hyperfine sublevels customarily numbered 1 through 6 . Normally states 1-6 are equally populated, and the polarization of a collection of atoms is zero. Each substate of deuterium, however, has non-zero polarization. To fully describe the nuclear polarization it is necessary to consider two types of polarization, vector and tensor. Table 1 shows polarization values for each sublevel when the magnetic field over the atoms is weak $\left(\mathrm{B} \ll \mathrm{B}_{\mathrm{c}}=117 \mathrm{G}\right)$. Typically $\mathrm{B}=5$-10G. Table 1 shows that a maximum tensor polarization, 1, is achieved by selecting atoms in either state 1 or state 4 . For reasons of intensity it is clearly advantageous to select both states 1 and 4 at the same time. In fact this is the optimal choice because no other states have positive tensor polarization. In most. applications it will be necessary to periodically reverse the sign of the target polarization. Again from Table 1 one can see that the optimal choice for negative tensor polarization is states 2 and 3. Similar arguments show that for the case of vector polarization one wants to alternate between states $1+6$ and states $4+5$.

\begin{tabular}{ccc} 
& Vector & Tensor \\
State 1 & 1 & +1 \\
State 2 & $1 / 3$ & -1 \\
State 3 & $-1 / 3$ & -1 \\
State 4 & -1 & +1 \\
State 5 & $-2 / 3$ & 0 \\
State 6 & $+2 / 3$ & 0 \\
\hline
\end{tabular}

TABLE 1. Values of vector and tensor polarization in the presence of a weak magnetic field for each of the six hyperfine sublevels of deuterium. 
In practice one wants to alternate between pairs $(1+4) \leftrightarrow(2+3)$ for tensor, and between $(1+6) \leftrightarrow(4+5)$ for vector in rapid succession. This can be accomplished by using so called RF transitions. While certain RF transitions were developed for application in polarized ion sources [so called weak field (WF) and strong field (SF) transitions], to produce the pairs of states required in a weak holding field a new type of "medium field" transition needs to be developed. RF transitions of this type have not previously been used for deuterium but some results have been reported for sodium. ${ }^{8-10}$ Table 2 shows combinations of RF transitions required for both vector and tensor polarized deuterium beams in a weak holding field. As indicated in the table the case of negative vector polarization requires that the MF transition operate as a WF. This is not technically difficult. The case of tensor polarization requires that the gradient of the static $B$ field in the transition unit change sign. This technique has not previously been reported. We have successfully constructed and tested a prototype MF transition unit for deuterium and have operated it in both iorward and reversed gradiants as required by this application.

\begin{tabular}{|c|c|c|c|c|c|}
\hline $\begin{array}{l}\text { Sign and Type } \\
\text { of Polarization }\end{array}$ & $\begin{array}{c}S F \\
3 \rightarrow 5\end{array}$ & $\begin{array}{c}\mathrm{SF} \\
2 \rightarrow 6\end{array}$ & $\begin{array}{c}\mathrm{MF} \\
(\text { new })\end{array}$ & $\begin{array}{c}\text { States } \\
\text { Selected }\end{array}$ & $\begin{array}{c}\text { Ideal } \\
\text { Polarization } \\
\text { Value }\end{array}$ \\
\hline Positive vector & on & on & off & $1+6$ & $+5 / 6$ \\
\hline Negative vector & on & on & $\begin{array}{c}\text { operate as } \\
\text { a WF transition }\end{array}$ & $4+5$ & $-5 / 6$ \\
\hline Positive tensor & on & off & $\begin{array}{c}\text { negative static } \\
\text { gradiant }\end{array}$ & $1+4$ & +1 \\
\hline Negative tensor & on & off & $\begin{array}{c}\text { positive static } \\
\text { gradient }\end{array}$ & $2+3$ & -1 \\
\hline
\end{tabular}

TABLE 2. Combinations of RF transitions required to produce positive ard negative vector and tensor polarization for a deuterium beam in a weak holding field.

The tests were conducted using the colliding beam ion source on the FN tandem at Wisconsin. When functioning properly the transition sequentially shifts the population of 
atoms in state 1 through states 2,3 and finally state 4 . The signature of this process is the appearance of regions of constant $P_{z}$ (vector polarization) between regions of changing $P_{z z}$ as a function of the static field strength.

Preliminary resulis at $18 \mathrm{MHz}$ are shown in Fig. 7. Better separation of these steps was observed at $24 \mathrm{MHz}$ and more tests are needed to accurately determine the transition probability now estimated to be $\geq 95 \%$. It is necessary to point out that the ideal polarization values in Fig. 7 apply only to the technique used to test the transition device. In actual use the ideal polarization values are those listed in Table 2.

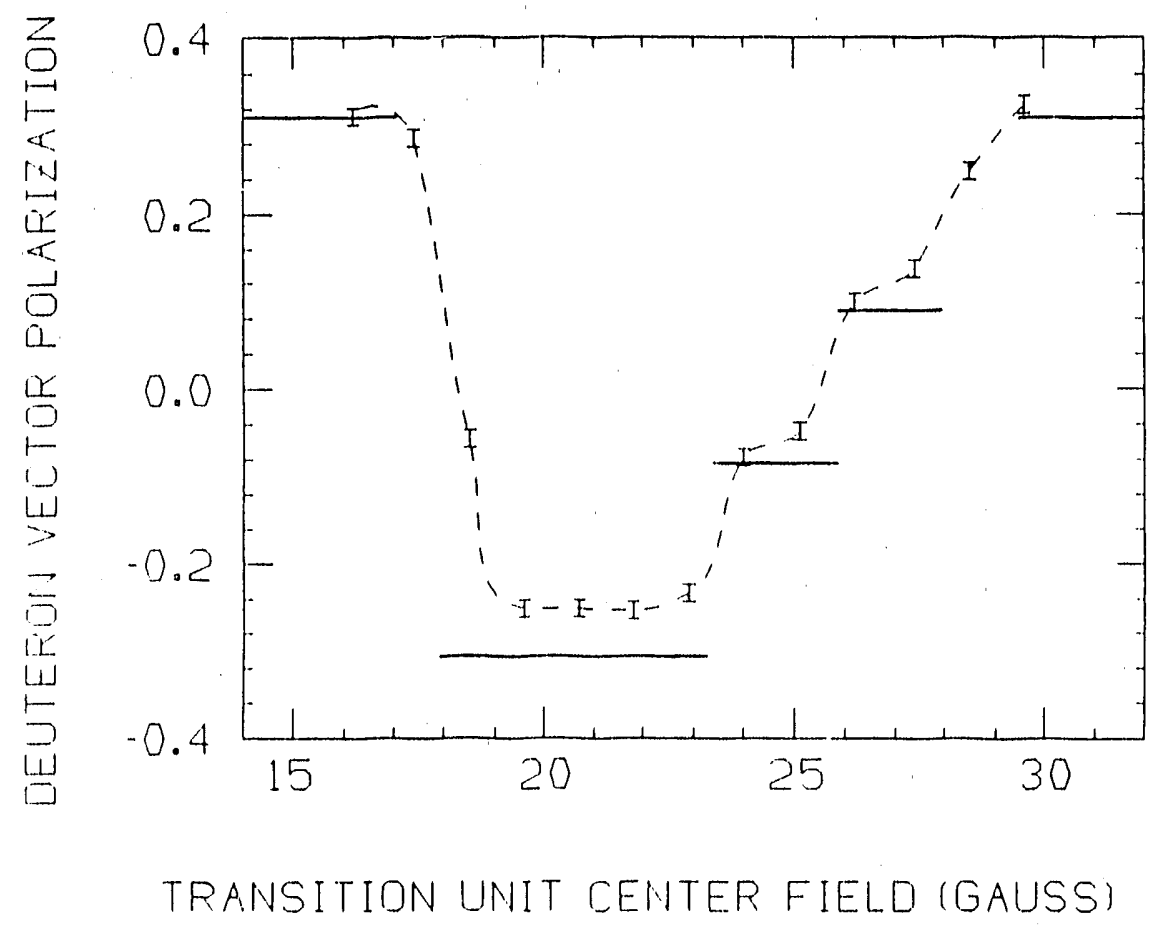

FIG. T. Preliminary results of MF transitions in deuterium at $18 \mathrm{MHz}$. Theoretical values for $P_{z}$ at each sequential step of the transition are shown as horizontal lines. The dashed line guides the eye.

This result greatly expands the options available to us in the final configuration of the atomic beam source. 


\section{PROPOSED RESEARCH}

\subsection{Strong Field Surface Tests}

We have completed most of the storage cell polarization tests in a weak magnetic field where the spins of the electron and the proton infuence one another. Depolarization is thought to take place through the interaction of the electron spin of the target atoms with the local field gradients on the storage cell wall, causing the target atom electron spin to flip. Low measured polarization values can also be due to recombination of atoms into molecules at the cell surface. We have succeeded in finding wall coatings that inhibit spinflip and recombination depolarization in weak field; these coatings will be used in storage ring applications where only small magnitude B-fields can be tolerated.

In situations where a strong magnetic field over the target cell is compatible with operation of the storage ring, one expects improved target polarization since electron spin-flip depolarization due to wall collisions would have a reduced effect on the spin orientation of the nucleus. It is expected that the reduction factor is of the order $\left(B / B_{c}\right)^{2}$, where $B_{c}$ is the "critical" field of the electron-nucleus interaction. This has yet to be demonstrated in nuclear spin polarized gas storage cell targets. Consequently, wall coatings that perform only moderately well in weak field tests could be satisfactory in strong field tests. For example, aluminum with a natural oxide layer shows significant loss in polarization after 380 wall collisions as compared to Teflon. But aluminum has advantages in other respects because of excellent high vacuum properties even at elevated temperatures, and because aluminum oxide is known to have good radiation resistance. We propose to study whether large target polarization is obtained with oxidized aluminum in a strong magnetic field. For construction of target cells with walls thin enough for recoils to exit through the wall of the cell, aluminum oxide is a very desirable material, since various laboratories (e.g. CERN) have developed methods to oxidize machined parts of aluminum and etching the unoxidized metal, to be left with a thin film of aluminum oxide, supported by a frame of (unetched) aluminum metal. Coatings that perform well in weak field tests, and have good vacuum properties, have high priority on the list of materials to be tested in a strong field.

Clurrently, work is being done on designing and fabricating the strong field coils, and new storage cell mounts that will accommodate the present cell refrigeration unit. It is of great interest to see if cooling the storage cell will result in a drop in polarization below 
$130 \mathrm{~K}$, as has been seen so far in every weak field test performed to date. A cell that can be cooled to $75 \mathrm{~K}$ without losing any polarization would yield a target denser by a factor of. two. High priority is being set on the task of developing a several $\mathrm{mA}$ output electron gun capable of being focused and aligned so as to permit injection of the needed beam current into the cell for ionization. The electrostatic extraction of the deuterium ions, $\mathrm{D}^{+}$, from the cell volume using a ramped axial magnetic field is a novel technique and will require significant development effort.

\subsection{Atomic Beam Sıurce}

We now have an operational, cooled but unfocussed atomic beam. This beam needs to be optimized for high intensity and degree of dissociation. We have constructed our dissociator so that the pyrex walls of the discharge 1egion can be cooled independently from the nozzle.

Preliminary results showed that an improvement in the degree of dissociation results from addition of water vapor. This needs to be restudied using the new capacitive coupling system. Other gases such as oxygen may also be helpful, and the dependence of degree of dissociation on concentration of gases adcled must be studied as a function of dissociator wall temperature. All of these parameters will interact somewhat with other factors such as flow rate, RF power level, and nozzle geometry. We will search for operating parameters that maximize the atomic flux entering the separation magnets.

Total beam intensity is not a complete characterization of the usefulness of an atomic beam. The beam intensity profile is also a crucial parameter because after passage through focusing magnets the beam must be injected into the feed pipe of a storage cell typically $1 \mathrm{~cm}$ dia $\times 10 \mathrm{~cm}$ long. The feed pipe also forms a leak path for stored atoms that reduce the target thickness. The size of this leak is proportional to $D^{3}$, where $D$ is the diameter of the pipe. At a constant total beam flux a smaller beam profile permits a smaller diameter feed pipe and therefore higher target thickness.

Measurement of neutral thermal atomic beam profiles is not easily accomplished. We previously attempted to measure atomic beam profile using sensitive semiconductor temperature sensors called "thermoflakes"11. The technique suffered from large drifts $i, 1$ the resistance of the sensors from oxygen depletion of the semiconductor material in the flakes. Recently glass coated versions of this material have become available. This may enable 
us to develop a reliable profile monitor. Samples have been obtained and a measurement technique is now under development.

We will monitor absolute beam intensity using the standard compression tube method but with a more accurate calibration method. The new method avoids the difficulty of mercury droplets in capillary tubes.

Once the prototype magnets have been assembled and installed we will use the above techniques to r.easure the resulting atomic beam properties. The observed absolute intensity and beam profile will be compared with computer generated trajectory calculations. These calculations are known to be incomplete because so far nobody has succeeded in including in the calculations the effects of gas scattering near the nozzle and inside the magnet bore. Nevertheless, the calculations should provide a useful guide in further magnet design. In this way an acceptable magnet geometry may be found within the next twelve months.

\subsection{Medium Field Transitions}

The preliminary results on the medium-field transitions shown in Sect. 2.4 suffer from a relatively large uncertainty in the overall normalization factor that results in a significant uncertainty in the transition probability for the device. Further measurements are needed to clarify the situation. We then intend to submit a paper to Nuclear Instruments and Methods for publication. For use in the atomic beam source, the medium-field transition devices must be designed and constructed for immersion in UHV vacuum. We also need to purchase a suitable power oscillator to drive the transitions.

In order to produce a fully functional atomic beam source two SF transitions must also be placed in the beam path as shown in Fig. 3. We have on hand two gold plated RF cavities from an older atomic beam device tuned to the required 3-5 and 2-6 transitions. They will need to be modified for UHV service. In addition all of the peripheral hardware associated with the transitions needs to be designed and constructed. In particular we will need RF power feedthroughs, magnet yokes to provide required field gradients, cooling of cavities, magnet windings, and RF power generators. 


\section{BUDGET}

We propose to contimue support for two Research Assistants in order to maintain reasonabl. progress on both phases of the program. As last year the Physicist and the two graduate students will be supported for 1-2 months of the year for other activities, since our budget does not permit full funding for 12 months. As before, we are not budgeting any expenditures for the Senior Investigator. The University is supporting the project by the academic year salary of the Senior Investigator and in the past has contributed one summer month's support for work on this project. Last year we obtained $\$ 20,090.00$ in equipment funds for this work from the University. We have recently applied for additional equipment funds. However, the funds available from the University this year are much more limited.

The amount budgeted for permanent equipment does not provide all the RF transition hardware but enough of it to test the basic components once the source becomes operational.

\section{REFERENCES}

1) T. Wise, A. Converse S. Price, High Energy Spin Physics, AIP Conf. Proc. 187 (AIP, New York NY 1989) p.1527.

2) P.G. Sona, Energie Nucleare 14, 295 (1967).

3) W. Mampe et al., Phys. Rev. Lett. 63, 593 (1989).

4) S.I. Mishnev et al., High Energy Spin Physics, AIP Conf. Proc. 187 (AIP, New York, NY 1989) p.1286.

5) H.F.Glavish, Nucl. Instrum. Methods 65, 1 (1968).

6) V.V. Afrosimov and N.V. Fedorenko, Sov. Phys. Tech. Phys. 2, 2391 (1958).

7) K. Halbach, Nucl. Instrum. Meth. 169, 1-10 (1980).

8) W. Dreves et al., Phys. Rev. Lett. 50, (22) 1759 (1883).

9) H. Jänsch, D. Krämer, E. Steffens, Hyperfine Interactions 22 253-260 (1985).

10) H. Jänsch et al., J. Phys. D 17, 231-237 (1984).

11) K.C. Harvey and C. Fehrenbach, Jr., Rev. Sci. Instrum. 54, (9) (1983). 


\section{PUBLICATIONS AND INVITED PAPERS SUPPORTED BY DOE $(1980 / 1990)$}

1. "Test of Storage for Polarized Atomic Hydrogen"

T. Wise, A. Converse, S. Price, High Energy Spin Physics; AIP Conference Proceęding No. 187 (AIP, New York, NY 1989) p.1527

2. "Summary Report: Polarized Gas Targets for Storage Rings", High Energy Physics, AIP Conference Proceedings No, 187 (AIP, New York, NY 1989), p.1487

3. "Internal Polarized Gas Targets for Storage Rings"

Invited Paper, W. Hacberli

American Physical Society, Baltimore, Maryland, May 1-4, 1989

4. "Preview and Review"

Invited Paper, W. Haeberli

1989 Topical Conference "Physics with Polarized Beams on Polarized Targets", University of Indiana, October 16-19, 1989

5. "Review of Physics with Polarized Beans and Polarized Gas Jets" Invited Paper, W. Haeberli

International Conference on "Polarized Beams and Polarized Gas Jets", KEK National Laboratory for High Energy Physics, Tsukuba, Japan, February 12-17, 1990

6. "Storage Cell Target for Polarized Hydrogen and Deuterium"

Invited Paper, W. Haeberli

Proceedings of the Ninth International Symposium on High Energy Spin Physics Bonn, Germany, 1990

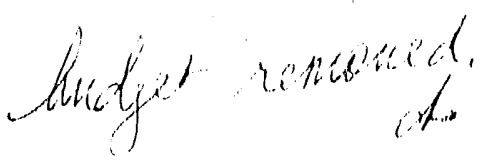



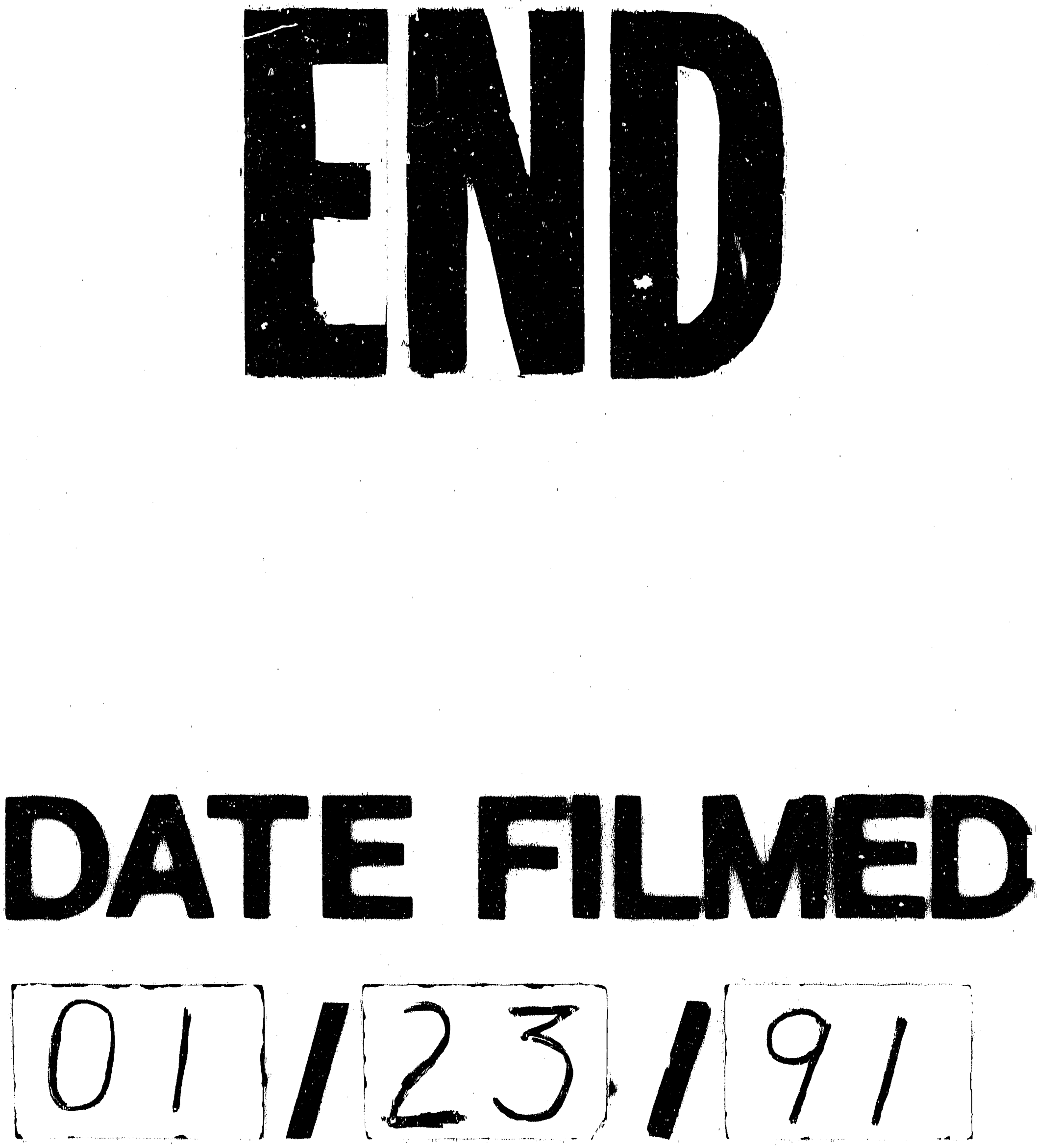
